\title{
Molecular evolution and genetics of postzygotic reproductive isolation in plants Andrea L. Sweigart ${ }^{1 *}$ and John H. Willis ${ }^{2 *}$
}

\author{
Addresses: ${ }^{1}$ Department of Genetics, University of Georgia, Athens, GA 30602, USA; ${ }^{2}$ Department of Biology, \\ Duke University, Durham, NC 27708, USA \\ *Corresponding authors: Andrea L. Sweigart (sweigart@uga.edu); John H. Willis (jwillis@duke.edu) \\ Fl000 Biology Reports 2012, 4:23 (doi:10.34I0/B4-23) \\ This is an open-access article distributed under the terms of the Creative Commons Attribution-Non Commercial License \\ (http://creativecommons.org/licenses/by-nc/3.0/legalcode), which permits unrestricted use, distribution, and reproduction in any medium, \\ provided the original work is properly cited. You may not use this work for commercial purposes. \\ The electronic version of this article is the complete one and can be found at: http://fl 000.com/prime/reports/b/4/23
}

\begin{abstract}
In just the last few years, plant geneticists have made tremendous progress in identifying the molecular genetic basis of postzygotic reproductive isolation. With more than a dozen genes now cloned, it is clear that plant hybrid incompatibilities usually evolve via two or more mutational steps, as is predicted by the Dobzhansky-Muller model. There is evidence that natural selection or random genetic drift can be responsible for these incompatibilities.
\end{abstract}

\section{Introduction}

The goal of explaining the origin of species has inspired more than two centuries of scientific inquiry, involving early naturalists through to modern evolutionary biologists. Though only hinted at by Darwin [1], the idea that the evolution of reproductive isolation is central to the process of speciation [2] is now widely recognized [3-5]. Reproductive isolating mechanisms that restrict gene exchange between diverging species include prezygotic barriers that limit the potential for mating or zygote formation (e.g. habitat and flowering time differences or pollen-pistil incompatibilities) and postzygotic barriers that reduce the viability or fertility of hybrid offspring if interbreeding does occur. Whereas the evolution of prezygotic isolation is often easily understood as a byproduct of differential adaptation to variable ecological conditions or of selection to reduce the production of low-fitness hybrids, the evolution of postzygotic isolation has been a longstanding mystery [1] because the production of dead or sterile hybrids cannot be favored by natural selection.

\section{The Dobzhansky-Muller model}

Dobzhansky [6] and Muller [7] outlined a solution to this puzzle, explaining that if postzygotic isolation is caused by incompatible gene interactions between diverging species then natural selection need not oppose its evolution.
A new mutation might function perfectly well in the context of its native genetic background, increasing in frequency until it becomes fixed within the species. But because the fitness of this new variant has only been "tested" against its own genetic background, it might be functionally incompatible with divergent alleles present in foreign genetic backgrounds, resulting in hybrid sterility or inviability. The key insight of the so-called DobzhanskyMuller model is that, if hybrid incompatibilities are caused by two or more mutational differences between species, there can be transitional genotypes that are adaptive or neutral in ancestral populations and, therefore, not eliminated by natural selection. In contrast, if reduced hybrid fitness is caused by a single mutational step (resulting in heterozygote disadvantage), natural selection is expected to impede the initial spread of the mutation within species. Nevertheless, in some situations, random genetic drift can overwhelm selection and lead to the fixation of such a mutation, particularly if the reduction in fitness is modest. Indeed, it is not uncommon for partial hybrid sterility in plants to be caused by heterozygous chromosomal rearrangements that evolved in a single mutational step $[4,8]$. Differentiating between these alternative genetic pathways to postzygotic isolation - one versus multiple mutational steps - can help identify which evolutionary processes contribute to species divergence. 
To determine whether hybrid incompatibilities evolve in multiple steps, as predicted by the Dobzhansky-Muller model, it is necessary to pinpoint the causal genetic changes at the molecular level. But even if the DobzhanskyMuller model is confirmed, it provides no expectation as to which kinds of molecular genetic changes cause postzygotic isolation. Are there predictable genetic pathways to incompatibilities? What types of molecular interactions cause hybrid dysfunction? The Dobzhansky-Muller model is also agnostic about the evolutionary processes that cause divergence within species. Do the initial mutations increase in frequency by random genetic drift or by natural selection because they benefit the native species for some reason that is incidental to their eventual contribution to reproductive isolation? In several plant systems, the molecular variants that cause hybrid inviability and sterility have recently been identified, providing some of the first hints at answers to these fundamental evolutionary questions. Here, we focus on exclusively nuclear-encoded hybrid incompatibility genes but point the reader to several excellent reviews of cytoplasmic male sterility $[9,10]$ and the population genetic theory for how cytonuclear incompatibilities might evolve [11,12].

\section{Recent advances}

\section{Two-locus hybrid incompatibilities}

In the classic model of speciation, hybrid incompatibilities are thought to evolve as a by-product of adaptation to different environments [13]. Some of the best evidence for this idea comes from recent work on hybrid necrosis, a form of plant hybrid inviability characterized by necrotic lesions, wilting, and inhibited growth [14]. A remarkable commonality has emerged among cloned hybrid necrosis genes: all encode proteins involved in plant defense against bacterial or fungal pathogens, and incompatible allelic combinations appear to induce autoimmune-like responses [15]. The plant immune system is a tightly coordinated network of proteins that recognize pathogen invasions and elicit a suite of cellular defense responses $[16,17]$. One might imagine that plant-pathogen molecular coevolution, occurring in independent populations with unique pathogen communities, could lead to hybrid mismatches between interacting components of the immune system that result in hyperactivation of defense responses in hybrid offspring. Indeed, two-locus incompatibilities between pathogen resistance genes and their interacting partners have been implicated in hybrid necrosis between divergent strains of Arabidopsis thaliana [18-20], between the indica and japonica subspecies of Asian cultivated rice [21], and between wild and domesticated species of tomato [22] and lettuce [23]. For a few of these loci, alleles that confer necrosis differ by several amino acid changes from ancestral alleles, perhaps consistent with a sustained fine-tuning of these proteins to improve pathogen recognition.

Other recent studies suggest that two-locus hybrid incompatibilities can also readily evolve in the absence of natural selection. Based on his early genetic analyses of hybrid sterility between the indica and japonica varieties of Oryza sativa, Oka suggested that defects in pollen development might be caused by loss-of-function alleles at different duplicate genes in each parent [24]. Postzygotic isolation might evolve passively then, due to degenerative mutations and genetic drift acting on different paralogs (genes related by duplication within a genome) in diverging populations $[25,26]$. Only recently has the cloning of several plant hybrid incompatibility genes provided empirical evidence for this idea. Indeed, hybrids between different strains of A. thaliana arrest as early embryos when they carry loss-offunction alleles at duplicate copies of the essential histidinol-phosphate amino-transferase gene ( $h p a 1$ and hpa2) [27]. Likewise, in two distinct Oryza crosses, null alleles at duplicate genes confer gametic male sterility $[28,29]$. Mizuta et al. [28] showed that pollen from indicajaponica hybrids fails to germinate when it carries loss-offunction alleles - one from each parent - at two duplicate genes of unknown function, DOPPELGANGER1 and 2 (DPL1 and DPL2). Similarly, pollen sterility in hybrids between $O$. sativa japonica and an Amazonian wild rice, Oryza glumaepatula, is caused by nonfunctional alleles at duplicate loci $S 27$ and $S 28$ that both encode the mitochondrial ribosomal L27 protein (mtRPL27) [29]. In this case, one lineage - O. glumaepatula - lacks the duplication; pollen is defective when it carries a loss-of-function allele at $S 28$ from $O$. sativa and is missing the $S 27$ paralog (by virtue of inheriting the corresponding genomic region from O. glumaepatula). An extraordinary feature of each of these systems is that loss-of-function alleles have evolved repeatedly. In $A$. thaliana, roughly three-quarters of plant collections carry one of six possible loss-of-function alleles at either hpa1 or hpa2 [27]. In Oryza, several different disruptive mutations have arisen in closely related species for both the DPL1/DPL2 and S27/S28 incompatibilities $[28,30]$. These findings, along with the rich history of whole genome duplication in plants, suggest that the divergent resolution of gene duplicates via mutation and genetic drift might be a common source of postzygotic isolation in plants.

\section{Single-locus hybrid incompatibilities}

In addition to the cases described above, studies have occasionally discovered hybrid incompatibilities that map to single genetic loci [31,32], which might be taken as prima facie evidence that postzygotic isolation can evolve via a single mutational step. Like the fixation of an underdominant chromosomal rearrangement, the establishment 
Figure I. The Dobzhansky-Muller model for a single-locus hybrid incompatibility

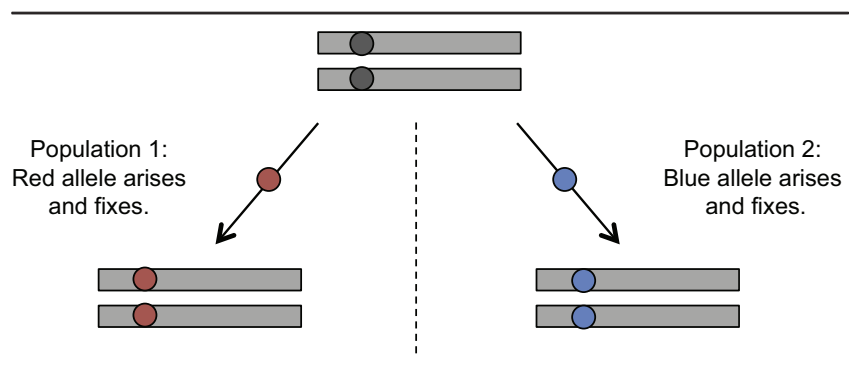

Red and blue alleles are incompatible in $\mathrm{F}_{1}$ hybrids

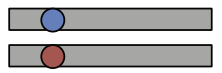

An ancestral population splits into two geographically isolated populations that diverge genetically and eventually fix different alleles (red or blue) at the same locus. In the $F_{1}$ hybrid, these two derived alleles are incompatible. Note that hybrid incompatibilities can just as easily arise between ancestral and derived alleles if two or more rounds of mutation and fixation occur within a lineage.

of a new sterility- or inviability-causing allele requires a hefty contribution from genetic drift to outweigh selection against unfit heterozygotes. But, if instead, a single-locus hybrid incompatibility is conferred by two or more alleles that evolve independently - implying multiple mutational steps - it is possible to circumvent any initial fitness valley within species [33] (Figure 1). The recent cloning of the single-locus hybrid incompatibility gene OAK [34], which encodes a receptor-like kinase (RLK) and causes reduced growth and developmental abnormalities in interpopulational hybrids of $A$. thaliana, supports just such a multiplestep scenario. Indeed, the two incompatible $O A K$ alleles are $9 \%$ divergent at the amino acid level, with 55 of 152 residues differing in a key malectin-like domain [34]. With such extreme levels of sequence diversity, it is reasonable to infer that intermediate, viable genotypes predated the current incompatible pair of alleles. Although its adaptive significance is unclear, the occurrence of $O A K$ within a highly variable tandem array of RLK genes suggests that high rates of gene conversion and illegitimate recombination may have contributed to its evolution [34].

The molecular characterization of three additional, single-locus incompatibilities between indica and japonica rice varieties also suggests the involvement of multiple mutational steps. The S5 incompatibility locus causes embryo sac abortion in indica-japonica heterozygotes, but certain "wide-compatibility" varieties carry neutral alleles and can, therefore, produce fertile progeny when crossed to either subspecies. Originally described by Ikehashi and
Araki as a simple, three-allele system [31], a reasonable assumption has been that the neutral allele represents an intermediate step that allowed the indica-japonica incompatibility to evolve without a fitness cost $[35,36]$. Upon closer inspection, however, genetic mapping of the S5 locus has revealed that hybrid female sterility is regulated by three, tightly linked genes - ORF3, ORF4, and ORF5 - and that haplotypes vary substantially among strains of indica, japonica, and wild rice $[37,38,39]$. During female sporogenesis, "killer" alleles at ORF4 and ORF5 cause endoplasmic reticulum stress that results in premature programmed cell death and embryo sac abortion, but these effects can be rescued by a "protector" allele at the adjacent ORF3 gene. This killer-protector combination appears to be the ancestral genotype and is at high frequency in the wild species Oryza rufipogon and Oryza nivara, as well as in the outgroup species O. glumaepatula [39]. In contrast, the typical indica S5 haplotype has a deletion in ORF4 that incapacitates its killing function, and the typical japonica haplotype carries mutations that disable both the ORF5 killer and ORF3 protector. The S5 incompatibility causes an aberrant, gain-of-function phenotype in indica-japonica hybrids that carry killer alleles at ORF4 and ORF5 but lack the protector allele at ORF3.

In the second case, Long et al. [40] fine-mapped the $S a$ locus to two distinct, tightly linked loci: SaM encodes a small ubiquitin-like modifier (SUMO) E3 ligase-like protein, and $\mathrm{SaF}$ encodes an F-box protein. Hybrid plants that are heterozygous at $S a M$ and also carry indica alleles at $S a F$ are semi-sterile because haploid pollen that inherits the japonica SaM allele fails to develop properly. To explain the surprising result that epistasis among three alleles is required for abortion of japonica SaM-carrying microspores, the authors propose a complicated model in which indica-encoded SaM and SaF proteins are transported between haploid microspores during early pollen development to kill gametes that inherit the japonica SaM allele [40]. The idea is that the japonica SaM protein is unable to move between microspores, so pollen grains that carry indica alleles at $S a M$ and $S a F$ - but do not receive the interacting japonica SaM protein - remain viable. For this incompatibility system to have evolved unopposed by natural selection, the japonica SaM allele must have spread after a "permissive" allele at SaF had fixed initially (Figure 2). Indeed, the current geographic distribution of $S a M / F$ haplotypes in O. sativa and its wild ancestor, O. rufipogon, is consistent with this scenario [40]. Moreover, patterns of SaM/F haplotype variation - particularly, the finding that japonica-like haplotypes segregate in $O$. rufipogon but not indica populations - support the hypothesis of independent origins of domestication for indica and japonica $[41,42]$. An important, unanswered question is what caused the japonica haplotype to become fixed. 
Figure 2. Model for the evolution of Sa hybrid sterility in rice
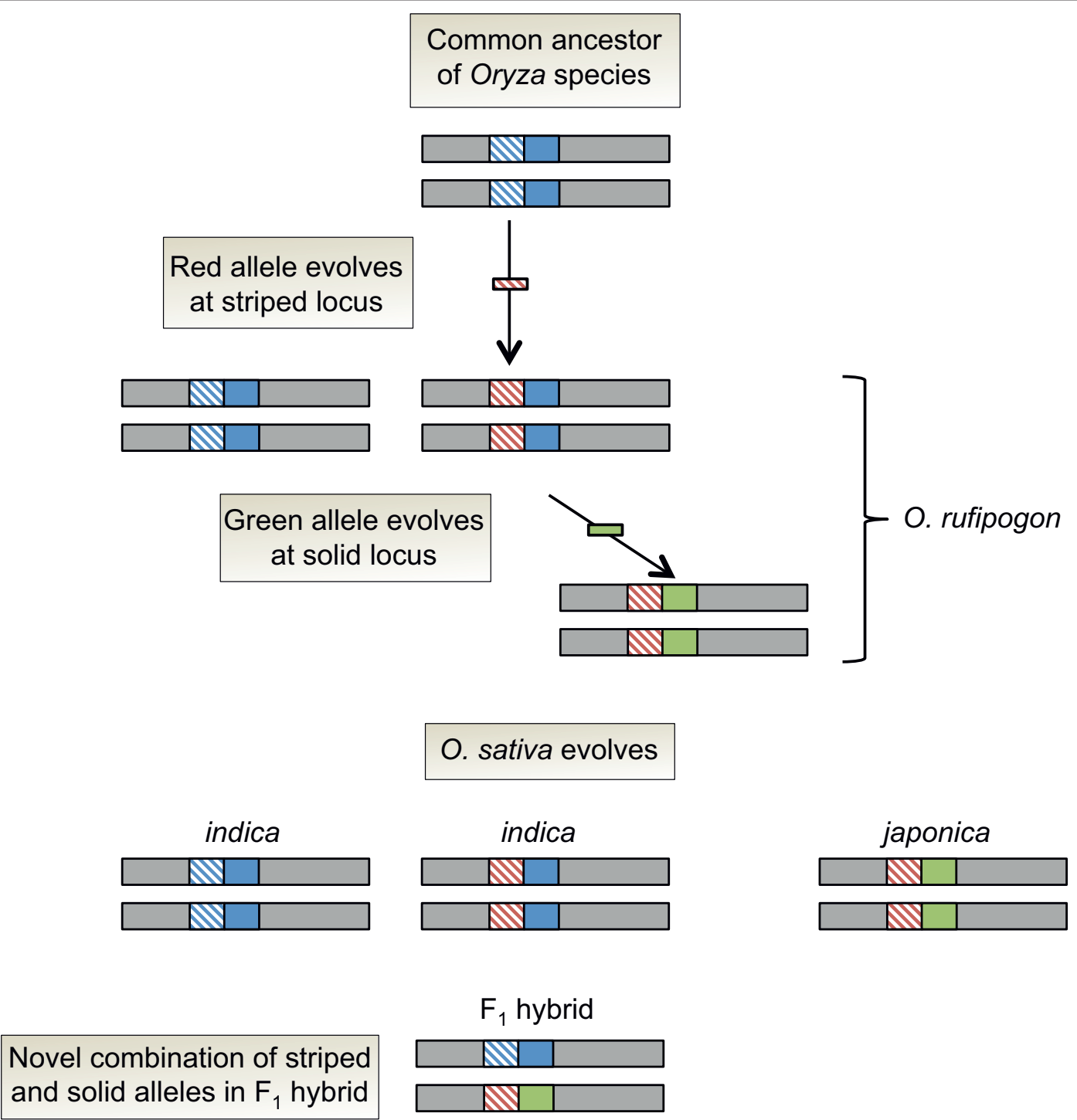

Sterility occurs when blue-encoded proteins are transported into microspores with green-encoded protein
A classic, single-locus incompatibility between Oryza sativa indica and Oryza sativa japonica is conferred by two adjacent genes (depicted here as striped and solid). Semi-sterility occurs in $F_{1}$ hybrids that carry blue alleles at the striped and solid genes (indica haplotype) in combination with a green allele at the solid gene (japonica haplotype). The wild progenitor Oryza rufipogon carries all three haplotypes: ancestral (blue, blue), "permissive” (red, blue), and japonica-like (red, green). Figure adapted from [40]. 
In the third case, Zhao et al. [43] mapped the S24 pollen sterility locus to an ankyrin-3 (ANK-3) gene, which Kubo et al. [44] later showed is dependent on an additional, unlinked locus, Epistatic Factor for S24(EFS). Like S5 and Sa, the S24-EFS hybrid incompatibility is a gain-of-function: pollen grains with japonica S24 alleles are aborted in S24-heterozygotes, but only when the plant is also homozygous for japonica alleles at EFS. As with $S a$, it is possible that the otherwise deleterious japonica S24 allele was only able to spread in a permissive, indica-like EFS background, but the necessary phylogeographic analyses have not yet been done. It is also worth noting that for both $S a$ and $S 24$, certain wide compatibility varieties carry neutral alleles that rescue pollen sterility $[43,45]$, indicating that the evolutionary histories of these incompatibilities might include alternate mutational routes.

\section{Conclusions and future challenges}

In just the last few years, plant geneticists have made tremendous progress in identifying the molecular genetic basis of postzygotic reproductive isolation. With more than a dozen genes now cloned, it is clear that plant hybrid incompatibilities usually evolve via two or more mutational steps, as is predicted by the DobzhanskyMuller model. This conclusion flows rather easily from those cases in which hybrid dysfunction is caused by epistasis between two or more unlinked genes, but it has been less obvious for a number of classic, single-locus incompatibilities [31]. Because of several recent detailed genetic studies, however, we now know that even these single-locus incompatibilities arose by mutations in two tightly linked genes, or, at the very least, two or more amino acid changes encoded within the same gene. Thus, it is possible that most of these incompatibility alleles appeared without any reductions in fitness within species.

Of course, the key question for speciation is which evolutionary forces allow incompatibility alleles to increase in frequency and eventually become fixed within species. For a few of the cases discussed above there is evidence that natural selection (e.g. hybrid necrosis) or random genetic drift (e.g. divergent resolution of duplicate genes) may have played a role. It also appears that there are some common genetic routes to plant hybrid dysfunction. Indeed, immune system genes seem to contribute disproportionately to hybrid necrosis. As we have seen, it is even possible for the very same gene to be involved in multiple cases of hybrid dysfunction: divergent duplicate loci carry a striking number of independently derived, loss-of-function mutations. Yet, there are many hybrid incompatibilities that do not involve disease resistance or duplicate genes, and, for these systems, we are only beginning to understand the evolutionary causes of divergence. Remarkably, at least 50 loci contribute to hybrid sterility between the closely related subspecies Oryza sativa indica and Oryza sativa japonica [46]. So does this large number reflect a special propensity in rice species for evolving hybrid sterility? Given that Oryza flowers have six anthers with many pollen grains per ovule, a gametic pollen killer that lowers fertility by $50 \%$ might entail little or no reduction in heterozygote seed fertility. Although our current sample of plant hybrid incompatibility genes comes from only two genera (Oryza and Arabidopsis), there are a number of classic [46,47] and more recently identified [48-51] incompatibility systems that are not yet molecularly defined. As advances in genomics and DNA sequencing technologies enable rigorous genetic analysis of reproductive isolation in a number of emerging model systems, we should gain new insight into which factors affect the number and nature of hybrid incompatibilities that accumulate between plant species.

\section{Abbreviations}

ANK-3, ankyrin-3; DPL, DOPPELGANGER; EFS, Epistatic Factor for S24; mtRPL27, mitochondrial ribosomal L27 protein; RLK, receptor-like kinase; SUMO, small ubiquitinlike modifier.

\section{Competing interests}

The authors declare that they have no competing interests.

\section{References}

I. Darwin C: On the Origin of Species. Murray: London; 1859.

2. Mayr E: Systematics and the Origin of Species. Columbia University Press: New York; 1942.

3. Schluter D, Conte GL: Genetics and ecological speciation. Proc Natl Acad Sci USA 2009, I 06(Suppl I):9955-62.

4. Coyne JA, Orr HA: Speciation. Sinauer: Sunderland, Massachusetts; 2004.

5. Sobel JM, Chen GF, Watt LR, Schemske DW: The biology of speciation. Evolution 2010, 64:295-315.

6. Dobzhansky T H: Genetics and the Origin of Species. Columbia University Press: New York; 1937.

7. Muller $\mathrm{HJ}$ : Isolating mechanisms, evolution, and temperature. Biol Symp 1942, 6:7 I- 125.

8. Rieseberg LH, Willis JH: Plant speciation. Science 2007, 317:910-4.

9. Chase CD: Cytoplasmic male sterility: a window to the world of plant mitochondrial-nuclear interactions. Trends Genet 2007, 23:8I-90.

10. Hanson MR, Bentolila S: Interactions of mitochondrial and nuclear genes that affect male gametophyte development. Plant Cell 2004, 16:SI54-69.

II. Hurst LD, Atlan A, Bengtsson BO: Genetic conflicts. $Q$ Rev Biol 1996, 71:317-64.

12. Burt A, Trivers R: Genes in Conflict: The Biology of Selfish Genetic Elements. Harvard University Press: Cambridge, MA; 2006.

13. Schluter D, Conte GL: Colloquium Papers: Genetics and ecological speciation. Proceedings of the National Academy of Sciences 2009, 106:9955-62. 
14. Bomblies K, Weigel D: Hybrid necrosis: autoimmunity as a potential gene-flow barrier in plant species. Nat Rev Genet 2007, 8:382-93.

\section{FlOOOPrime}

\section{RECOMMENDED}

15. Bomblies K: Doomed lovers: mechanisms of isolation and incompatibility in plants. Annu Rev Plant Biol 2010, 61:109-24.

\section{FlOOOPrime} RECOMMENDED

16. Jones JDG, Dangl JL: The plant immune system. Nature 2006, 444:323-9.

17. Dodds PN, Rathjen JP: Plant immunity: towards an integrated view of plant-pathogen interactions. Nat Rev Genet 2010, I I:539-48.

18. Bomblies K, Lempe J, Epple P, Warthmann N, Lanz C, Dangl JL, Weigel D: Autoimmune response as a mechanism for a Dobzhansky-Muller-type incompatibility syndrome in plants. PLoS Biol 2007, 5:e236.

\section{FlOOOPrime \\ RECOMMENDED}

19. Alcázar R, García AV, Parker JE, Reymond M: Incremental steps toward incompatibility revealed by Arabidopsis epistatic interactions modulating salicylic acid pathway activation. Proc Natl Acad Sci USA 2009, 106:334-9.

\section{FlOOOPrime}

20. Alcázar R, García AV, Kronholm I, de Meaux J, Koornneef M, Parker JE, Reymond M: Natural variation at Strubbelig Receptor Kinase 3 drives immune-triggered incompatibilities between Arabidopsis thaliana accessions. Nat Genet 20 I0, 42: I I35-9.

\section{FlOOOPrime}

21. Yamamoto E, Takashi T, Morinaka Y, Lin S, Wu J, Matsumoto T, Kitano H, Matsuoka M, Ashikari M: Gain of deleterious function causes an autoimmune response and Bateson-DobzhanskyMuller incompatibility in rice. Mol Genet Genomics 2010, 283:305-15.

\section{FloOOPrime}

\section{RECOMMENDED}

22. Krüger J, Thomas CM, Golstein C, Dixon MS, Smoker M, Tang S, Mulder L, Jones JDG: A tomato cysteine protease required for Cf-2-dependent disease resistance and suppression of autonecrosis. Science 2002, 296:744-7.

\section{FlOOOPrime}

\section{RECOMMENDED}

23. Jeuken MJW, Zhang NW, McHale LK, Pelgrom K, den Boer E, Lindhout P, Michelmore RW, Visser RGF, Niks RE: Rin4 causes hybrid necrosis and race-specific resistance in an interspecific lettuce hybrid. Plant Cell 2009, 21:3368-78.

\section{FlOOOPrime \\ RECOMMENDED}

24. Oka $\mathrm{H}$ : Analysis of genes controlling $\mathrm{f}(\mathrm{I})$ sterility in rice by the use of isogenic lines. Genetics 1974, 77:52 I-34.

\section{FlOOOPrime}

\section{RECOMMENDED}

25. Lynch M, Force A: The probability of duplicate gene preservation by subfunctionalization. Genetics 2000, 154:459-73.

\section{FlOOOPrime}

26. Werth CR, Windham MD: A Model for Divergent, Allopatric Speciation of Polyploid Pteridophytes Resulting from Silencing of Duplicate-Gene Expression. The American Naturalist I991, 137:5I5-26.

27. Bikard D, Patel D, Le Metté C, Giorgi V, Camilleri C, Bennett MJ, Loudet O: Divergent evolution of duplicate genes leads to genetic incompatibilities within A. thaliana. Science 2009 323:623-6.

FIOOOPrime

28. Mizuta $Y$, Harushima $Y$, Kurata N: Rice pollen hybrid incompatibility caused by reciprocal gene loss of duplicated genes. Proc Natl Acad Sci USA 2010, 107:20417-22.

\section{FlOOOPrime}

\section{RECOMMENDED}

29. Yamagata $Y$, Yamamoto E, Aya K, Win KT, Doi K, Sobrizal, Ito T, Kanamori H, WuJ, Matsumoto T, Matsuoka M, Ashikari M, Yoshimura A: Mitochondrial gene in the nuclear genome induces reproductive barrier in rice. Proc Natl Acad Sci USA 2010, 107:1494-9.

\section{FlOOOPrime
RECOMMENDED}

30. Win KT, Yamagata Y, Miyazaki Y, Doi K, Yasui H, Yoshimura A: Independent evolution of a new allele of $F I$ pollen sterility gene S27 encoding mitochondrial ribosomal protein L27 in Oryza nivara. Theor Appl Genet 20II, I 22:385-94.

31. Sano Y: The genic nature of gamete eliminator in rice. Genetics 1990, I25:183-91.

\section{FlOOOPrime \\ RECOMMENDED}

32. Ikehashi $\mathrm{H}$, Araki $\mathrm{H}$ : Genetics of FI sterility in remote crosses of rice. In Rice Genetics. Edited by IRRI. Philippines International Rice Research Institute: 1986:119-30.

33. Nei M, Maruyama T, Wu Cl: Models of evolution of reproductive isolation. Genetics 1983, 103:557-79.

34. Smith LM, Bomblies K, Weigel D: Complex evolutionary events at a tandem cluster of Arabidopsis thaliana genes resulting in a single-locus genetic incompatibility. PLoS Genet 201I, 7:e1002164.

\section{FlOOOPrime RECOMMENDED}

35. Nei M, Nozawa M: Roles of mutation and selection in speciation: from Hugo de Vries to the modern genomic era. Genome Biology and Evolution 20 I I, 3:8I 2-29.

36. Rieseberg LH, Blackman BK: Speciation genes in plants. Annals of Botany 2010, 106:439-55.

37. Chen J, Ding J, Ouyang Y, Du H, Yang J, Cheng K, Zhao J, Qiu S, Zhang X, Yao J, Liu K, Wang L, Xu C, Li X, Xue Y, Xia M, Ji Q, Lu J, $\mathrm{Xu}$ M, Zhang Q: A triallelic system of S5 is a major regulator of the reproductive barrier and compatibility of indica-japonica hybrids in rice. Proc Natl Acad Sci USA 2008, 105: I |436-41.

\section{FlOOOPrime} RECOMMENDED

38. Du H, Ouyang $\mathrm{Y}$, Zhang $\mathrm{C}$, Zhang Q: Complex evolution of S5, a major reproductive barrier regulator, in the cultivated rice Oryza sativa and its wild relatives. New Phytol 201 I, 191:275-87.

\section{FlOOOPrime}

RECOMMENDED

39. Yang J, Zhao X, Cheng K, Du H, Ouyang Y, Chen J, Qiu S, Huang J, Jiang $Y$, Jiang L, Ding J, Wang J, Xu C, Li X, Zhang Q: A killerprotector system regulates both hybrid sterility and segregation distortion in rice. Science 2012, 337:1336-40.

\section{FlOOOPrime
RECOMMENDED}

40. Long Y, Zhao L, Niu B, Su J, Wu H, Chen Y, Zhang Q, Guo J, Zhuang C, Mei M, Xia J, Wang L, Wu H, Liu Y: Hybrid male sterility in rice controlled by interaction between divergent alleles of two adjacent genes. Proc Natl Acad Sci USA 2008, 105:1887I-6.

\section{FlOOOPrime}

RECOMMENDED

4I. Londo JP, Chiang Y, Hung K, Chiang T, Schaal BA: Phylogeography of Asian wild rice, Oryza rufipogon, reveals multiple independent domestications of cultivated rice, Oryza sativa. Proc Natl Acad Sci USA 2006, 103:9578-83. 
42. Sang $T$, Ge S: The puzzle of rice domestication. Journal of Integrative Plant Biology 2007, 49:760-8.

43. Zhao ZG, Zhu SS, Zhang YH, Bian XF, Wang Y, Jiang L, Liu X, Chen LM, Liu SJ, Zhang WW, Ikehashi H, Wan JM: Molecular analysis of an additional case of hybrid sterility in rice (Oryza sativa L.). Planta 20I I, 233:485-94.

44. Kubo $\mathrm{T}$, Yoshimura $\mathrm{A}$, Kurata $\mathrm{N}$ : Hybrid male sterility in rice is due to epistatic interactions with a pollen killer locus. Genetics 20II, I89:I083-92

45. Wang $Y$, Zhong ZZ, Zhao ZG, Jiang L, Bian XF, Zhang WW, Liu LL, Ikehashi $\mathrm{H}$, Wan JM: Fine mapping of a gene causing hybrid pollen sterility between Yunnan weedy rice and cultivated rice (Oryza sativa L.) and phylogenetic analysis of Yunnan weedy rice. Planta 2010, $231: 559-70$.

46. Ouyang $Y$, Liu $Y$, Zhang $Q$ : Hybrid sterility in plant: stories from rice. Curr Opin Plant Biol 2010, 13:186-92.

47. Rick CM: Abortion of male and female gametes in the tomato determined by allelic interaction. Genetics 1966, 53:85-96.
48. Moyle LC, Graham EB: Genetics of hybrid incompatibility between Lycopersicon esculentum and L. hirsutum. Genetics 2005, 169:355-73.

\section{FloOPrime}

RECOMMENDED

49. Macnair MR, Christie P: Reproductive isolation as a pleiotropic effect of copper tolerance in Mimulus guttatus. Heredity 1983, 50:295-302.

50. Sweigart AL, Fishman L, Willis JH: A simple genetic incompatibility causes hybrid male sterility in mimulus. Genetics 2006 , I72:2465-79.

FlOOPrime

RECOMMENDED

5I. Burkart-Waco D, Josefsson C, Dilkes B, Kozloff N, Torjek O, Meyer R, Altmann T, Comai L: Hybrid incompatibility in Arabidopsis is determined by a multiple-locus genetic network. Plant Physiol 2012, I58:80I-I2. 